\title{
The Role of Diagnostic Hysterolaparoscopy in the Evaluation of Infertility in Tertiary Care Centers
}

\author{
Ali El-shabrawy Ali, Sherin Attia Shazly Essa, Ahmed Metwally Mohmoud Al Ktway, \\ Remma Mahmoud Ali Soulah \\ Department of Obstetrics and Gynecology, Faculty of Medicine, Zagazig University, Egypt \\ *Correspondence to: Remma Mahmoud Ali Soulah, Mobile: (+20) 01002132769, E-mail: soulah81@ gmail.com
}

\begin{abstract}
Background: Female infertility constitutes one of the commonest problems in gynecological practice. Infertility is defined as failure to achieve pregnancy during one year of frequent unprotected intercourse.

Objective: This study aimed to evaluate the role of the combined laparoscopy and hysteroscopy in the diagnosis and treatment of infertile female.

Patients and methods: This retrospective study was conducted at Gynecologic Endoscopic Unit, Zagazig University Hospital during the period from December 2020 to May 2021. Two hundred infertile women were included in this study. They were divided by the following percent 1:1 primary to secondary infertile cases. Group I: $50 \%$ patients with primary infertility and group II included 50\% patients with secondary infertility. Women aged 20-40 years with normal hormone profile without male factor infertility were included.

Results: The frequency of women with arcuate uterus is significantly increased in group I than in group II. Regarding frequency of women with arcuate uterus, bicornuate and very small uterine cavity were significantly increased in group I than in group II and the frequency of women with intrauterine synechia and bilateral narrow corneal end were significantly higher in group II than in group I.

Conclusions: laparoscopy and hysteroscopy play very important role as diagnostic and therapeutic tools in the infertile women. Combined diagnostic simultaneous laparoscopy and hysteroscopy should be performed in all infertile patients before the treatment.
\end{abstract}

Keywords: Hysteroscopy; infertility; laparoscopy.

\section{INTRODUCTION}

Infertility is defined in specific terms as failure to conceive after one year of unprotected regular intercourse. A broader view of infertility includes, not being able to carry a pregnancy to term and have a baby (1). Infertility affects about $10 \%-15 \%$ of couples of childbearing ages. A malfunction in the fallopian tubes is responsible for $40-50 \%$ of cases, while uterine problems are estimated at about $15 \%$ of cases and other factors include an ovulation defect of 20-30\% of cases. Laparoscopic findings showed endometriosis in majority of primary infertility and polycystic ovarian syndrome (PCOS) in majority of secondary infertility patients along with other pathologies ${ }^{(2)}$.

Laparoscopic surgery, is a minimally invasive surgery (MIS), band aid surgery, or keyhole surgery, is a modern surgical technique in which operations in the abdomen are performed through small incisions (usually $0.5-1.5 \mathrm{~cm}$ ) as opposed to the larger incisions needed in laparotomy. Keyhole surgery displays images on TV monitors to magnify the surgical elements. Laparoscopic surgery includes operations within the abdominal or pelvic cavities. It belongs to the broader field of endoscopy ${ }^{(3)}$.

Hysteroscopy is a well-established diagnostic and operative technique. Hysteroscopy procedures are highly appreciated mainly for their minimal invasiveness, suitability for office gynecology, cost effectiveness and safety ${ }^{(4)}$.
Combined laparoscopy and hysteroscopy is considered the gold standard for evaluation of causes of infertility; as the advantages of combined hysteroscopy and laparoscopic approach is proper assessment of the distal tubes and ovaries, peritoneal cavity, and the elimination of tubal spasm as a factor of infertility, absence of radiation, more precise application of instruments and confirmation of achievement of tubal patency during the procedure ${ }^{(5)}$.

The aim of this study was to evaluate the role of the combined laparoscopy and hysteroscopy in the diagnosis and treatment of infertile female.

\section{PATIENTS AND METHODS}

This retrospective study was conducted at Gynecologic Endoscopic Unit, Zagazig University Hospital during the period from December 2020 to May 2021. It included two hundred infertile women aged between 20 and 40 years old. who were divided into two groups: Group I, 50\% patients with primary infertility and group II that included 50\% patients with secondary infertility.

Inclusion criteria: All fulfilled the following criteria: Primary or secondary infertility of more than 1 year duration. Primary infertility patients were those who had never conceived before, while secondary infertile patients had at least one prior conception, irrespective of the outcome. Ultrasonic folliculometry was suggestive of ovulation. 
Normal hormonal profiles for infertility e.g. (serum FSH, LH). Normal thyroid function and prolactin level. Normal semen analysis.

Exclusion Criteria: Women under 20 or above 40 years old. Hormonal abnormalities known to cause anovulation like thyroid dysfunction. Hyperprolactinemia. Polycystic ovarian syndrome. Couples with abnormal semen analysis. Women having any contra-indication for laparoscopy as chronic chest disease, cardiac disease and marked obesity.... etc. Women who have marked adhesions suggested by history. Suspicion of pregnancy, to avoid the possibility of disrupting an implanting gestation, so patients are examined in the early proliferative phase of the cycle. Symptoms suggestive of pelvic or lower genital tract infection, to avoid exacerbating the symptoms. Patients with advanced or uncontrolled medical disease e.g. DM or rheumatic fever or T.B.

All patients were subjected to the following: History taking from patient records. Physical examination from patient records: complete physical examination including (blood pressure, height, weight, thyroid examination, breast examination and bimanual examination). Male partner is evaluated by urologist for congenital anomalies, testicular......etc.

Female partner has so many factors to be evaluated for infertility including the following factors: Ovarian factor, pelvic adhesion, bilateral tubal obstruction, uterine factor, cervical factor and vaginal factor.

Investigation of infertility has been observed including (semen analysis for her husbanded, serum F.S.H, L.H, serum prolactin level, ovulation assessment and hysterosalpingography ...etc.). Transvaginal ultrasound and folliculometry. Routine preoperative investigations were done $(\mathrm{CBC}$, random blood sugar, liver function, kidney function, PT, PTT, INR, ECG, Viral markers HBV, HCV, HIV, and urine analysis).

\section{Ethical approval:}

The study was approved by the Research Ethical Committee of Faculty of Medicine, Zagazig University. The work was carried out for studies involving humans in accordance with the World Medical Association's Code of Ethics (Helsinki Declaration).

\section{Hysteroscopy surgical technique:}

The patient was placed in the dorsal lithotomy position. The thighs should be at a 90 degree angle to the table in order to create enough space for the surgeon to manipulate the hysteroscopy. The patient perineum should be just out the edge of the table. Normal saline was used for uterine distension connected to the inflow channel on the sheath with intravenous tubing. A vaginal disinfection with a non-irritating watery disinfection solution was performed without placing speculum. Before the hysteroscopy and sheath were inserted into the external os, the sheath was flushed to remove the air. The tip of the hysteroscopy was positioned in the vaginal introitus, the labia being slightly separated with fingers. The vagina was distended with saline. The scope was driven to the posterior fornix to readily visualize the portio and slowly backwards to identify the external cervical os. When this became visible, the scope was carefully moved forward to the internal os to follow the black spot to internal os and toward uterine cavity, titled 30 degree trying not to touch side walls to be pain free and with least possible trauma.

The uterine cavity was systematically explored by rotating the fore-oblique scope in order to identify any anomaly in the uterine walls and/or the right and left tubal ostia. Systematic examination of all four walls of the uterine cavity and the tubal openings was carried out with axial movements of the telescope. The endometrium is smooth and pink white in color during the proliferative phase and lush and velvety in the secretory phase. Any abnormal pathology was documented.

Finally, the evaluation and finding data were written in details by the surgeon and the technique of the procedure was done according to the surgeon evaluation and patient condition. Any complications in the form of pain, bleeding, vasovagal attack and perforation, were registered in the patient sheet.

\section{Laparoscopic surgical technique:}

The patient is placed in a supine position with abduction of lower limbs and with flexion of the thighs onto the pelvis of about $20^{\circ}$. This position allows concomitant abdominal and vaginal access without the need to change the position of the patient. In order to avoid injuries of the brachial plexus, the two arms are positioned alongside the body. The placement of the lower limbs should avoid compression of the sciatic nerve, external popliteal nerve, and calves. The buttocks of the patient should project slightly beyond the edge of the operating table to facilitate the uterine manipulation.

Classically, pneumoperitoneum is insufflated using the Veress needle placed at the Palmer's point (left hypochondrium, 2-3 cm below the costal margin, at the mid clavicular line). At this level, pneumoperitoneum creation is easy even in obese patients

After the skin incision, a $10 \mathrm{~mm}$ trocar was placed inside the umbilicus for the zero-degree laparoscope. Systematically, we used three ancillary trocars: two 5 $\mathrm{mm}$ trocars for the main surgeon and one $5 \mathrm{~mm}$ trocar for the assistant surgeon. The two lateral trocars were placed about $2 \mathrm{~cm}$ medial to the anterior-superior iliac spine (and always lateral to the inferior epigastric vessels), and the third trocar was placed infra umbilical, in the midline, about $8-10 \mathrm{~cm}$ below the umbilical trocar. 


\section{Statistical Analysis}

Statistical analysis was done using IBM@ SPSS $\odot$ Statistics version 22 (IBM@ Corp., Armonk, NY, USA) and MedCalc@ version 13 (MedCalc@ Software bvba, Ostend, Belgium). Data were expressed as mean \pm standard deviation for quantitative variables and as number and percentage for qualitative ones. The chisquare, ANOVA and paired t tests were used for interpretation of results. A P-value of $\leq 0.05$ was considered statistically significant.

\section{RESULTS}

Table (1): Demographic data

\begin{tabular}{|c|c|c|c|c|c|c|}
\hline & \multicolumn{2}{|c|}{ Group I } & \multicolumn{2}{|c|}{ Group II } & $\mathbf{p}$ & $\mathbf{t}$ \\
\hline \begin{tabular}{|l} 
Age (years) \\
$X \pm$ SD range
\end{tabular} & \multicolumn{2}{|c|}{$\begin{array}{c}25.7 \pm 3.8 \\
18-35\end{array}$} & \multicolumn{2}{|c|}{$\begin{array}{c}30.1 \pm 3.3 \\
24-38\end{array}$} & 0.001 & 6.05 \\
\hline $\begin{array}{l}\text { Duration of infertility (years) } \\
X \pm \text { SD range }\end{array}$ & \multicolumn{2}{|c|}{$\begin{array}{c}4.5 \pm 2.8 \\
1-13\end{array}$} & \multicolumn{2}{|c|}{$\begin{array}{l}5.86 \pm 2.6 \\
2-13\end{array}$} & 0.012 & 2.5 \\
\hline \multirow[t]{2}{*}{ Laparoscopic findings } & \multicolumn{2}{|c|}{$\begin{array}{l}\text { Group I } \\
\mathbf{N}=100\end{array}$} & \multicolumn{2}{|c|}{$\begin{array}{c}\text { Group II } \\
\mathrm{N}=100\end{array}$} & \multirow[t]{2}{*}{$\mathbf{X}^{2}$} & \multirow[t]{2}{*}{$\mathbf{P}$} \\
\hline & $\mathbf{N}$ & $\%$ & $\mathbf{N}$ & $\%$ & & \\
\hline \begin{tabular}{|l|} 
Abnormal findings \\
\end{tabular} & 14 & 14.0 & 12 & 12.0 & 0.09 & 0.76 \\
\hline
\end{tabular}

Table (1) showed that age of women and duration of infertility were significantly lower in group I compared to group П. Abnormal findings by laparoscopy were comparable between two groups.

Table (2): Uterine laparoscopic findings in both groups

\begin{tabular}{|c|c|c|c|c|c|c|}
\hline \multirow{2}{*}{ Laparoscopic findings } & \multicolumn{2}{|c|}{$\begin{array}{c}\text { Group I } \\
\mathbf{N}=100\end{array}$} & \multicolumn{2}{|c|}{$\begin{array}{c}\text { Group II } \\
\mathbf{N}=\mathbf{1 0 0}\end{array}$} & \multirow[t]{2}{*}{$\mathbf{P}$} & \multirow[t]{2}{*}{$\mathbf{X}^{2}$} \\
\hline & $\mathbf{N}$ & $\%$ & $\mathbf{N}$ & $\%$ & & \\
\hline $\begin{array}{c}\text { Abnormal uterine findings } \\
\text { Arcuate uterus } \\
\text { Subserous fibroid }\end{array}$ & $\begin{array}{l}4 \\
3\end{array}$ & $\begin{array}{l}4.0 \\
3.0\end{array}$ & $\begin{array}{c}2.0 \\
4\end{array}$ & $\begin{array}{l}2.0 \\
4.0\end{array}$ & $\begin{array}{l}5.21 \\
0.09\end{array}$ & $\begin{array}{c}0.02 * \\
0.15\end{array}$ \\
\hline
\end{tabular}
in group $\Pi$.

Table (2) showed that the frequency of women with arcuate uterus was significantly increased in group I than

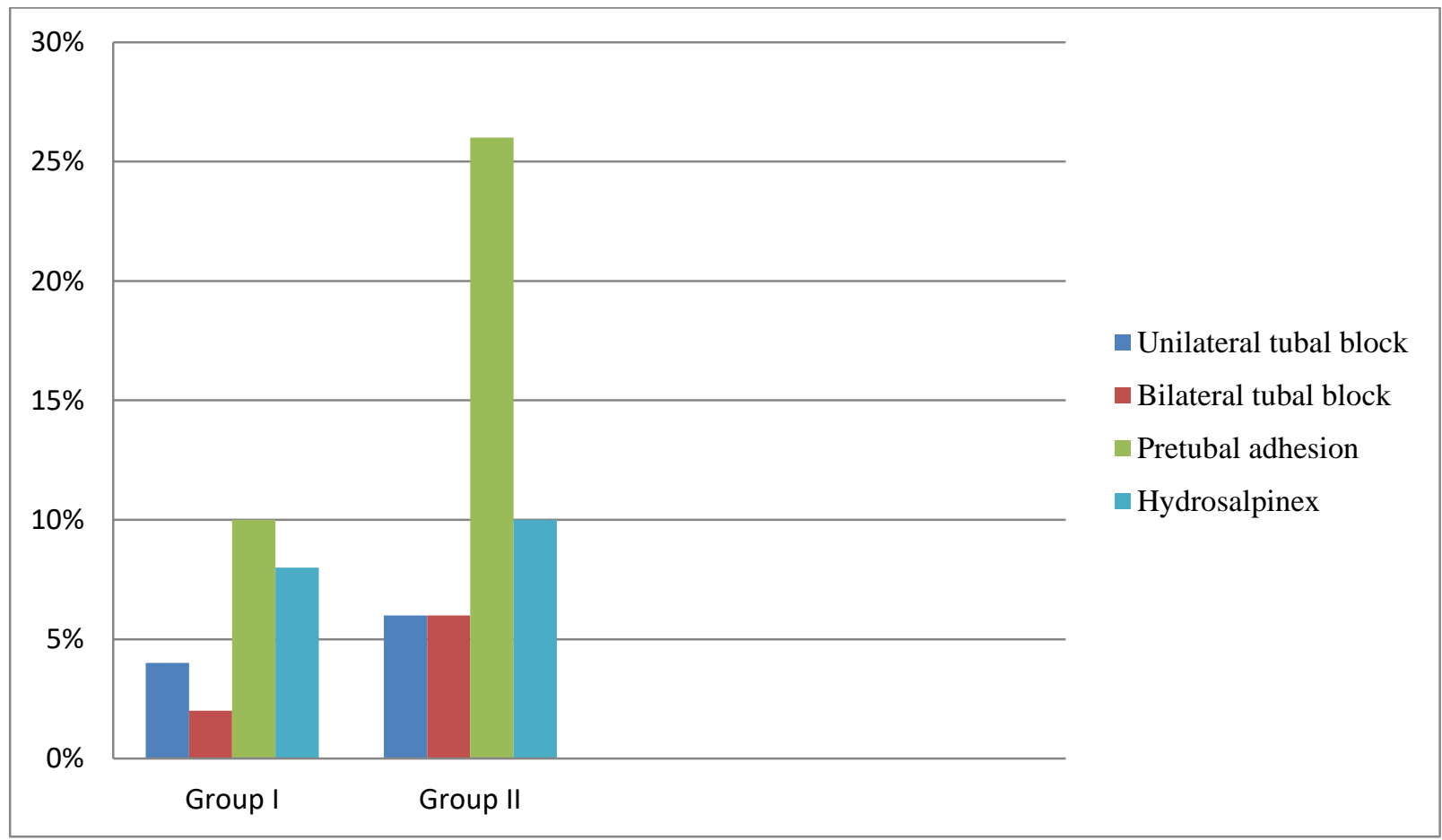

Figure (1): Tubal laparoscopic findings in both groups

Figure (1) showed that the frequency of women with bilateral distal tubal block in group $\Pi$ was more than in group I. 
Table (3): Peritoneal laparoscopic findings in both groups

\begin{tabular}{|c|c|c|c|c|c|c||}
\hline \multirow{2}{*}{ Laparoscopic findings } & \multicolumn{2}{|c|}{$\begin{array}{c}\text { Group I } \\
\text { N=100 }\end{array}$} & \multicolumn{2}{c|}{$\begin{array}{c}\text { Group I } \\
\text { N=100 }\end{array}$} & \multirow{2}{*}{ P } & \multirow{2}{*}{$\mathbf{X}^{\mathbf{2}}$} \\
\cline { 2 - 5 } & $\mathbf{N}$ & $\mathbf{\%}$ & $\mathbf{N}$ & $\boldsymbol{\%}$ & & \\
\hline \hline Endometriosis & 5 & 5.0 & 3 & 3.0 & $0.005^{*}$ & 1.0 \\
\hline
\end{tabular}

* Significance

Table (3) showed that there was significant difference among the frequency of women with endometriosis in both groups being more in group 1.

Table (4): Surgical procedure by laparoscope among the two groups

\begin{tabular}{||c|c|c|c|c|c|c||}
\hline \multirow{2}{*}{ Laparoscopic procedure } & \multicolumn{2}{|c|}{$\begin{array}{c}\text { Group I } \\
\text { N=100 }\end{array}$} & \multicolumn{2}{c|}{$\begin{array}{c}\text { Group } \mathbf{~ I} \\
\text { N=100 }\end{array}$} & \multirow{2}{*}{ P } & \multirow{2}{*}{$\mathbf{X}^{\mathbf{2}}$} \\
\cline { 2 - 5 } & $\mathbf{N}$ & $\mathbf{\%}$ & $\mathbf{N}$ & $\mathbf{\%}$ & & \\
\hline \hline Milking of tubes & 3.0 & 3 & 6 & 6.0 & $.005^{*}$ & 0.0 \\
\hline Sub serous myomectomy & 0.0 & 0 & 1 & 2.0 & 1.0 & 0.0 \\
\hline Open laparotomy & 0.0 & 0 & 1 & 2.0 & 1.0 & 0.0 \\
\hline Pelvic adhesiolysis & 6.0 & 6 & 7 & 7.0 & 1.33 & 7.17 \\
\hline Linear salpingostomy & 1.0 & 1.0 & 3 & 3.0 & $.002^{*}$ & 0.0 \\
\hline salpingectomy & 2.0 & 2.0 & 4 & 4.0 & $.004^{*}$ & 0.0 \\
\hline
\end{tabular}

* Significance

N.B: In some patients more one procedure was done

Table (4) showed that there were significant differences as regards tubal milking, linear salpingotomy and salpingectomy significantly increased in group $\Pi$ than in group I.

Table (5): Hysteroscopy findings in both groups

\begin{tabular}{|l|c|c|c|c|c|c||}
\hline \multirow{2}{*}{\multicolumn{1}{|c|}{ Hysteroscopic findings }} & \multicolumn{2}{|c|}{$\begin{array}{c}\text { Group I } \\
\text { N=100 }\end{array}$} & \multicolumn{2}{c|}{$\begin{array}{c}\text { Group I } \\
\text { N=100 }\end{array}$} & \multirow{2}{*}{$\mathbf{X}^{2}$} & \multirow{2}{*}{ P } \\
\cline { 2 - 6 } & $\mathbf{N}$ & $\mathbf{\%}$ & $\mathbf{N}$ & $\mathbf{\%}$ & & \\
\hline \hline Normal findings & 58 & 58.0 & 48 & 48.0 & 00.0 & 1.0 \\
\hline Arcuate uterus & 4 & 4.0 & 0 & 0.0 & 5.21 & $0.02^{*}$ \\
\hline Subseptate uterus & 6 & 6.0 & 2 & 2.0 & 1.89 & $0.033^{*}$ \\
\hline Bicornuate uterus & 5 & 5.0 & 0 & 0.0 & 4.13 & $0.04^{*}$ \\
\hline Infantile uterine cavity & 4 & 4.0 & 0 & 0.0 & 5.1 & $0.02^{*}$ \\
\hline Submucou smyoma & 0 & 0.0 & 8 & 8.0 & 0.0 & $0.001^{*}$ \\
\hline Intrauterine synechia & 0 & 0.0 & 7 & 7.0 & 4.54 & $0.04^{*}$ \\
\hline Single endometrium polyp & 7 & 7.0 & 6 & 6.0 & 3.33 & 1.1 \\
\hline Multiple endometrium polyp & 3 & 3.0 & 0 & 0.0 & 1.0 & $0.012^{*}$ \\
\hline Atrophic endometrium & 3 & 3.0 & 2 & 2.0 & 0.0 & 1.0 \\
\hline Hypertrophic endometrium & 3 & 3.0 & 8 & 8.0 & 1.04 & 0.8 \\
\hline Bilateral narrow corneal ends & 3 & 3.0 & 10 & 10.0 & 6.07 & $0.001^{*}$ \\
\hline Unilateral narrow corneal end & 4 & 4.0 & 9 & 9.0 & 0.54 & $0.06^{*}$ \\
\hline
\end{tabular}

* Significance 
Table (5) showed that the frequency of women with arcuate uterus, bicornuate uterus and very small uterine cavity were significantly increased in group I than in group II. The frequency of women with intrauterine synechia and bilateral narrow corneal end were significantly higher in group $\Pi$ than in group I.

\section{DISCUSSION}

The results of this study showed that the mean age of patients in group one was $25.7 \pm 3.8$ and of group two was $30.1 \pm 3.3$ years where the age of group one was significantly lower than the age of group two. The result also showed that the mean of duration of infertility in group one was $4.5 \pm 2.8$, but the mean of duration of infertility in group two was $5.86 \pm 2.9$ years. So, the mean of duration of infertility was significantly lower in group one compared to group two. This comes in sgreement with Haider et al. ${ }^{(6)}$, who reported that out of 200 subfertile patients total 30 patients were selected for laparoscopy. $20(66 \%)$ patients were in primary infertility group while $10(33 \%)$ patients were in secondary infertility group. $11(55 \%)$ patients of primary infertility belonged to age group of $18-25$ years while $6(60 \%)$ patients of secondary infertility belonged to age group of 26-33 years. Mean duration of subfertility at time of presentation in primary infertility group was 1.95 years while in secondary infertility was 2.70 years.

The current study showed that, laparoscopic findings in group one was abnormal in 14 (14\%) women and in group two was abnormal in $12(12 \%)$ women, this means that there is no significant difference regarding abnormal laparoscopic findings in the two groups. This disagrees with Shetty ${ }^{(7)}$, who reported that 50 infertile women underwent laparoscopy during the study period, 32 (64\%) had primary infertility while $18(36 \%)$ had secondary infertility, $8(25 \%)$ patients with primary and $2(11.1 \%)$ patients with secondary had no visible abnormality.

In this study, the frequency of women with arcuate uterus in group one was 4 (4\%), but was 2 in group two. This means that the frequency of women with arcuate uterus is significantly higher in group one than in group two. This is in agreement with Chan et al. ${ }^{(5)}$, who reported that, the prevalence of uterine anomalies diagnosed by laparoscopy was $8.0 \%$ in infertile women, $13.3 \%$ in those with a history of miscarriage and $24.5 \%$ in those with miscarriage and infertility.

In this study, the frequency of women with endometriosis in group one was $5(5 \%)$ and in group two was $3(3 \%)$. This means that there was significant difference between the two groups being higher in primary infertility group. This is in agreement with Haider et al. ${ }^{\left({ }^{6}\right)}$ who reported that 30 patients were selected for laparoscopy. $20(66 \%)$ patients were in primary infertility group while $10(33 \%)$ patients were in secondary infertility group. The most common cause observed in patients with primary infertility, was endometriosis spots, which accounted for 11 (55\%) with associated symptoms (dysmenorrhea, dyspareunia and irregular cycles). In secondary infertility tubal occlusion was more common, which accounted for 3 $(30 \%)$.

In this study, tubal laparoscopic findings in both groups were defined as the frequency of women with peritubal adhesion. In group one, there were $2(2 \%)$ and in group two, there were 4 (4\%). This means that the peritubal adhesion was significantly higher in group two than in group one. This is in agreement with Shetty (7) who reported that pelvic inflammatory disease (PID) was found in $1(3.1 \%)$ and $2(16.7 \%)$ cases of primary and secondary infertility respectively. Peritubal adhesions were detected in $2(6.3 \%)$ cases with primary infertility and $4(22.2 \%)$ cases with secondary infertility. Also, Hu et al. ${ }^{(8)}$ reported that there was no significant difference in the percentage of uni- and bilateral tubal patency cases between the two groups $(69.4 \%$ versus $68.9 \%)$. There was significant difference in the percentage of pelvic adhesions in primary infertility cases and in secondary infertility cases (42.9\%, versus $60.7 \%$ ). Similar to ours, Bhandari et al. (9) reported endometriosis and adnexal adhesions as the commonest abnormalities detected at laparoscopy evaluation of 546 patients with infertility. In addition, Poncelet et al. ${ }^{(10)}$ reported endometriosis and pelvic adhesions as the commonest laparoscopic findings in their 114 patients with infertility.

According to our results with abnormal laparoscopic findings in studied patients, laparoscopic evaluation of these patients is a useful tool in the workup of those patients with both diagnostic and therapeutic benefits. In this study, the laparoscopic surgical procedures were as follows: the frequency of pelvic adhesiolysis in group one was $6(6 \%)$ but in group two, it was 7 (7\%). This means that pelvic adhesiolysis was higher in group two than in group one but with no statistically significant difference. This is against Farquhar et al. ${ }^{(\mathbf{1 1})}$ who reported that there was no evidence of a difference in occurrence and ongoing pregnancies between laparoscopic adhesolysis. There was no evidence of a difference in miscarriage rates between the two groups.

In this study, hysteroscopy findings were normal in 58\% in group one and $48 \%$ in group two. This is not in agreement with Elbareg et al. ${ }^{(12)}$ who reported that out of 200 patients with infertility in whom standard infertility investigations were normal who underwent hysteroscopy evaluation 135 (67.5\%) patients showed normal uterine cavity, while abnormal cavity was detected in $65(32.5 \%)$ of patients. Intrauterine adhesions were $7 \%$ in second group and no cases detected in first group. This is not in agreement with Elbareg et al. ${ }^{(12)}$ who reported mild endometrial adhesions in their patients with infertility $(28 / 200,14 \%)$. 
Single endometrial polyp was $7 \%$ in the first group and $6 \%$ in the second group. Endometrial polyps impair endometrial receptivity as evidenced by lower endometrial HOXA 10, and HOXA 11 receptivity markers in patients with endometrial polyps. Shobha $\boldsymbol{e t}$ al. (13) reported that endometrial polyp was the commonest hysteroscopy uterine abnormality representing $10.13 \%$ and $19.05 \%$ in 100 women with primary and secondary infertility respectively.

Both groups, were defined as the frequency of arcuate uterus, in group one was 4 but in group two was 0 and the frequency of bicornate uterus in group one was 5 but in group two was 0 . This means that the previous congenital uterine anomalies were significantly higher in group one than in group two. While, the frequency of women with intrauterine synechiae was 0 in group one but was 7 in group two and the frequency of women with bilateral narrow corneal ends was 3 in group one but was 10 in group two. This means that intrauterine synechiae and bilateral narrow corneal ends were significantly increased in group two than in group one.

\section{CONCLUSIONS}

Combined laparoscopy and hysteroscopy is important in evaluating the infertile women where the combined procedures achieve shortening of the investigation time, proper assessment of the distal tubes and ovaries, elimination of spasm as a factor, absence of radiation, more precise application of instruments and confirmation of achievement of tubal patency during the procedure.

\section{REFERENCES}

1. Gurunath S, Pandian Z, Anderson $\mathrm{R}$ et al. (2018): Defining infertility-a systematic review of prevalence studies. Hum Reprod., 17 (5): 575-78.
2. Sutaria $R$, Singh $R$, Gopal $S$ (2020): Hysterolaparoscopy as a one-time approach in infertility: a prospective study. Int J Reprod Contracept Obstet Gynecol., 9 (7): 2992-7.

3. Walid M, Heaton $R$ (2011): Laparoscopy-tolaparotomy quotient in obstetrics and gynecology residency programs. Arch Obestet Gynecol., 283 (5): 1027-1031.

4. Abbott J, Garry R (2012): Hysteroscopic surgery. Hum Reprod., 8 (1): 68-78.

5. Chan Y, Jayaprakasan K, Thomton J (2011): The prevalence of congenital uterine anomalies in unselected and high-risk population. Hum Reprod., 17 (6): 761-71.

6. Haider J, Rani S, Talpur S (2018): Laparoscpic evaluation of female fertility. J Ayub Med Coll Abbottabad, 22 (1): 136-8.

7. Shetty S, Shetty H, Rai S (2013): Laparoscopic evaluation of tubal factor in cases of infertility. Int $\mathbf{J}$ Reprod Contracept Obstet Gynecol., 2 (3): 410-13.

8. Hu X, Wang D, Caie W (2020): Application of fertiloscopy in infertile women. Zhonghua Fu Chan Ke Za Zhi., 40 (12): 840-3.

9. Bhandari S, Singh A, Agarwal P et al. (2015): Findings in diagnostic laparoscopy in patients with unexplained infertility. Fertil Sci Res., 2: 29-33.

10. Poncelet $\mathbf{C}$, Bonneau $C$, Sifer $C$ et al. (2019): Interest of laparoscopy for (unexplained infertility).Fertil Steril., 96: 148-49.

11. Farquhar C, Lilford R, Marjoribanks J (2015): Laparoscpic drilling by diathermy or laser for ovulation induction in anovulatory polycystic ovary syndrome. Cochrane Database Syst Rev., 20 (3): 1122-26.

12. Elbareg M, Fathi M, Kamal A et al. (2019): Value of hysteroscopy in management of unexplained infertility. Asian Pacific Journal of Reproduction, 3 (4): 295-298.

13. Shobha D, Madhy K, Amiti A (2019): Role of diagnostic hysterolaparoscopy in evaluation of primary and secondary infertility. Journal of Evolution of Medical and Dental Science, 3: 2194-2207. 